BRIEF COMMUNICATION

\title{
Evaporation-induced hydrodynamics promote conjugation- mediated plasmid transfer in microbial populations
}

\author{
Chujin Ruan ${ }^{1,2}$, Josep Ramoneda ${ }^{2}$, Guowei Chen $\mathbb{D i D}^{3}$, David R. Johnson (iD) ${ }^{凶}$ and Gang Wang (iD) ${ }^{\text {网 }}$
}

(c) The Author(s) 2021

Conjugative plasmids bestow important traits to microbial communities, such as virulence, antibiotic resistance, pollutant biotransformation, and biotechnology-relevant functions. While the biological mechanisms and determinants of plasmid conjugation are well established, the underlying physical and ecological driving forces remain unclear. Microbial communities often inhabit unsaturated environments, such as soils and host surfaces (e.g., skin, teeth, leaves, roots), where water evaporation and associated small-scale hydrodynamic processes frequently occur at numerous air-water and solid-water interfaces. Here, we hypothesized that evaporation can induce water flows with profound effects on the spatial distribution and surface deposition of cells, and consequently on the extent of plasmid conjugation. Using droplet experiments with an antibiotic resistance-encoding plasmid, we show that evaporation-induced water flows reduce cell-cell distances and significantly increase the extent of plasmid conjugation. Counterintuitively, we found that evaporation results in lower expression levels of conjugation-related genes. This negative relationship between the extent of plasmid conjugation and the expression of conjugation-related genes could be attributed to increased conjugation efficiency during evaporation. This study provides new insights into the physical and ecological determinants of plasmid conjugation, with important implications for understanding the spread and proliferation of plasmidencoded traits.

ISME Communications (2021)1:54 ;https://doi.org/10.1038/s43705-021-00057-5

\section{MAIN}

Conjugative plasmids confer important traits to microbial communities, with both deleterious and beneficial effects on human health, the environment, and biotechnology [1-3]. The spread of virulence and resistance to antimicrobial agents $[1,2]$ and the facilitation of specific pollutant biotransformations exemplify the importance of conjugative plasmids [3, 4]. Understanding the mechanisms governing the transfer and spread of conjugative plasmids is therefore critically important. Although substantial research efforts have been made toward understanding the molecular mechanisms and biological determinants of plasmid conjugation [5], the underlying driving forces from physical and ecological aspects remain unclear.

Many microbial communities exist in environments that are periodically or continuously exposed to unsaturated water conditions. For example, the communities residing in the vadose zone of soils are periodically exposed to saturated conditions after rainfall events and irrigation, and thereafter to unsaturated conditions upon soil draining. The microbial communities inhabiting the outer surfaces of various hosts such as skin, teeth, leaves, or roots also experience frequent hydration dynamics. The air-water interfaces of such soil particles or host surfaces are subject to water evaporation when the ambient relative humidity $(\mathrm{RH})$ is $<100 \%$. How does small-scale water evaporation modulate plasmid conjugation within these microbial communities?
At air-water interfaces, evaporation usually induces localized water flows that can be a driving force for passive cell movement, and thereby redistributes cells across space and affects their surface deposition [6]. Notably, the distances via evaporationinduced passive cell movement are often longer than those attributed to active cell movement (e.g., via flagella or pili) [7]. Hence, evaporation-induced passive cell movement may remarkably contribute to the spatial redistribution and surface deposition of cells in unsaturated environments. Accordingly, we hypothesize that evaporation is an important determinant of the number and duration of cell-cell contacts, and thus an important determinant of plasmid conjugation [5].

We tested this hypothesis using droplet experiments that allowed us to control evaporation-induced passive cell movement. We used the bacterium Escherichia coli HB101 carrying the IncP atype broad-host-range conjugative plasmid RP4 as the donor strain, where RP4 encodes for ampicillin (Amp), kanamycin, and tetracycline (Tet) resistance [8]. We used E. coli K12 carrying plasmid pNW33n as the recipient strain, where pNW33n is a nonconjugative plasmid that encodes for chloramphenicol (Chl) resistance and green fluorescent protein (GFP) [9]. Successful conjugation of RP4 thus results in recipient cells that express GFP and are resistant to all four antibiotics. We performed droplet experiments by mixing the donor and recipient at equal concentrations and depositing $2 \mu \mathrm{l}$ liquid droplets of the mixture

\footnotetext{
${ }^{1}$ College of Land Science and Technology, China Agricultural University, 100193 Beijing, China. ${ }^{2}$ Department of Environmental Microbiology, Swiss Federal Institute of Aquatic Science and Technology (Eawag), 8600 Dübendorf, Switzerland. ${ }^{3}$ School of Civil and Hydraulic Engineering, Hefei University of Technology, Hefei, China. email: david.johnson@eawag.ch; Gangwang@cau.edu.cn
} 

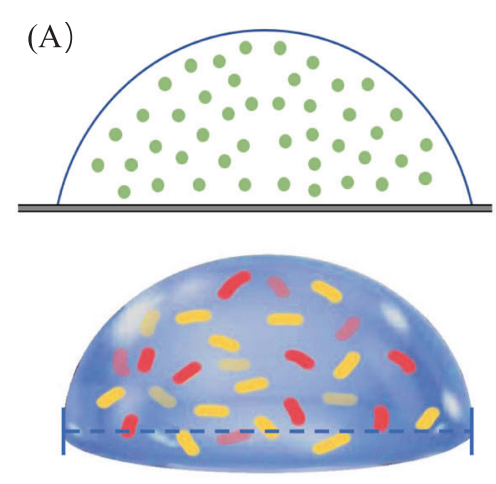

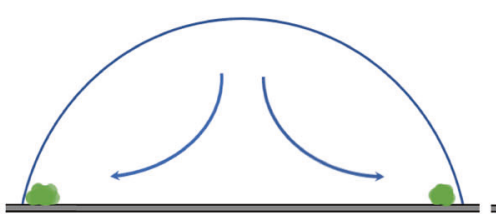

Coffee ring effect

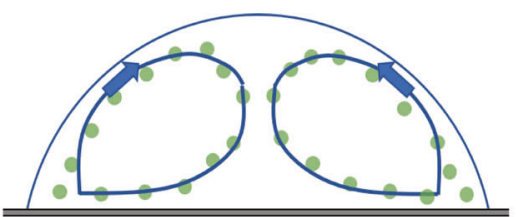

Marangoni effect

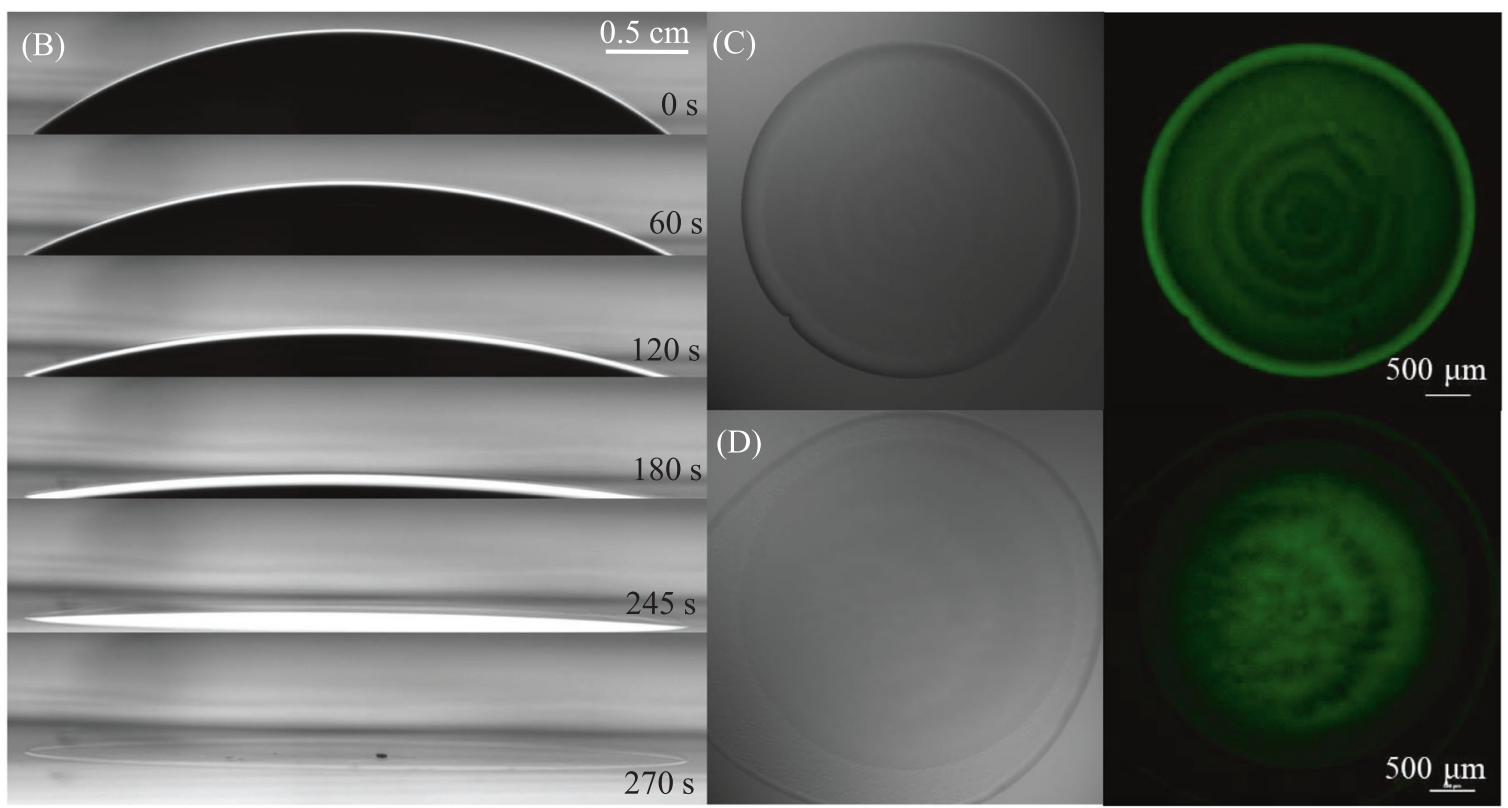

Fig. 1 Effects of evaporation-induced water flow on passive cell movement and spatial redistribution. A Schematic representation of the coffee-ring effect and Marangoni effect and their depositional model. The dark blue arrows represent the direction of the flow field caused by evaporation under the coffee-ring effect and Marangoni effect. The red and yellow cells represent the donor and recipient that spatially redistribute under different conditions. B Experimental time-lapse droplet evaporation with a pinned air-water-solid interface on an agar surface. C The final experimentally observed spatial distribution of E. coli K12 cells (GFP) after evaporation with the coffee-ring effect. D The final experimentally observed spatial distribution of $E$. coli K12 cells (GFP) after evaporation with the Marangoni effect counteracting the coffee-ring effect.

in different environments. We incubated the droplets under nonevaporative (NEV; placing the droplets into $1.5 \mathrm{ml}$ microcentrifuge tubes with $100 \% \mathrm{RH}$ ), evaporative (EV; placing the droplets on agar plates and incubating them in a constant humidity chamber at $30 \% \mathrm{RH}$ ), and Marangoni convection (MC; placing the droplets on agar plates containing $1 \%$ polyethylene glycol (PEG) and incubating the plates in a constant humidity chamber at $30 \% \mathrm{RH}$ ) conditions. We expected that water flow would not be observed and cells would remain dispersed throughout the droplets under NEV conditions, while uneven evaporation would drive water and cells toward the perimeter (i.e., the'coffee-ring' effect) due to capillary forces under EV conditions (Fig. 1A) [10]. Under MC conditions, we expected centripetal Marangoni flows to counteract the capillary-driven movement of water and cells toward the perimeter (Fig. 1A) [11, 12]. We tracked changes to the droplets using a Drop Shape Analyzer DSA25I (A. Krüss Optronic, Hamburg, Germany) and imaged the droplets using a Nikon A1RsiHD 25 confocal laser scanning microscope (Nikon, Tokyo, Japan) (see details in the Supplementary Information). We performed all of our experiments three times for each treatment (i.e., three droplets per treatment), with three analytical replicates per droplet. We further demonstrated that there is no loss in cell viability over the time-course of the experiments (Supplementary Table S1).

We found that incubation conditions (NEV, EV, and MC) indeed have a profound effect on the spatial distribution of cells within the droplets (Fig. 1 and Supplementary Fig. S1). For NEV conditions, cells remained dispersed throughout the droplets for the duration of the experiment. For EV conditions, the droplets rapidly evaporated (Fig. 1B) and cells were transported to the solid-liquid-air interface, resulting in concentrated cells at the perimeter that formed a biofilm-like mass (Fig. 1C and Supplementary Fig. S1). We did not detect active flagellar motility under EV conditions, indicating that cells were likely transported via evaporation-induced passive movement (Supplementary Video 1). For MC conditions, the addition of PEG altered the trajectories of cells, with a portion moving toward the solid-liquid-air interface at the perimeter and another returning to the center of the droplets as expected by centripetal Marangoni flows (Supplementary Video 2) [11, 12]. Overall, PEG successfully inhibited the 'coffeering' effect and led to more uniform spatial distributions of cells within the droplets (Fig. 1D and Supplementary Fig. S1).

We also found that incubation conditions (NEV, EV, and MC) had a profound effect on the extent of RP4 conjugation (Fig. 2 and 
(A)

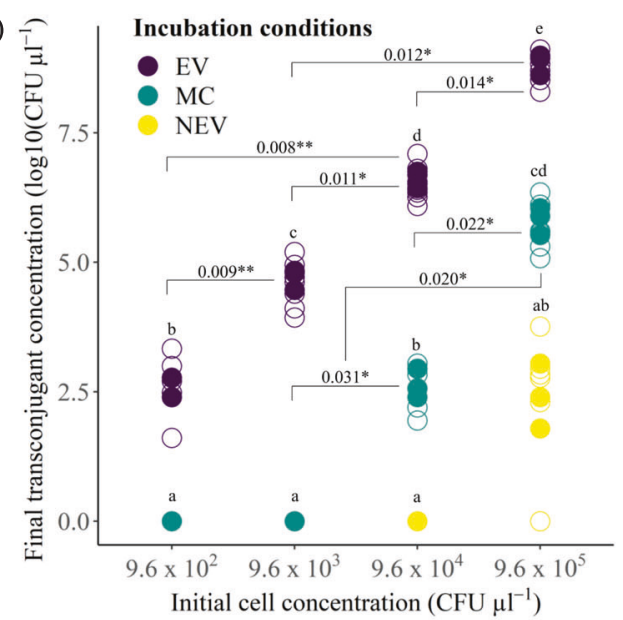

(C)

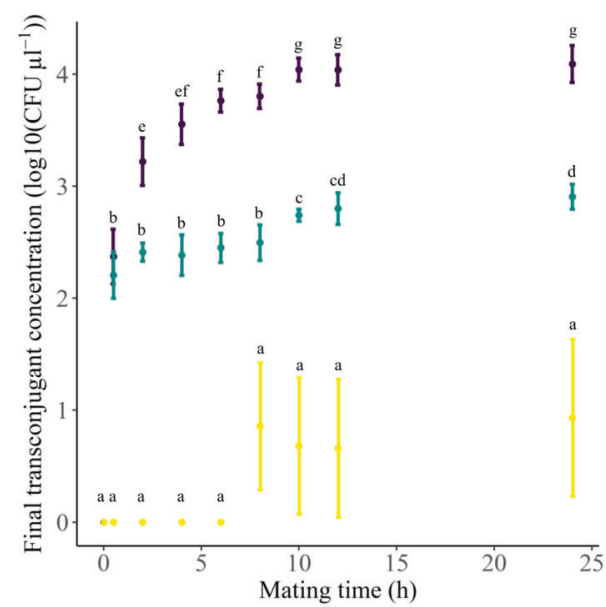

(B)

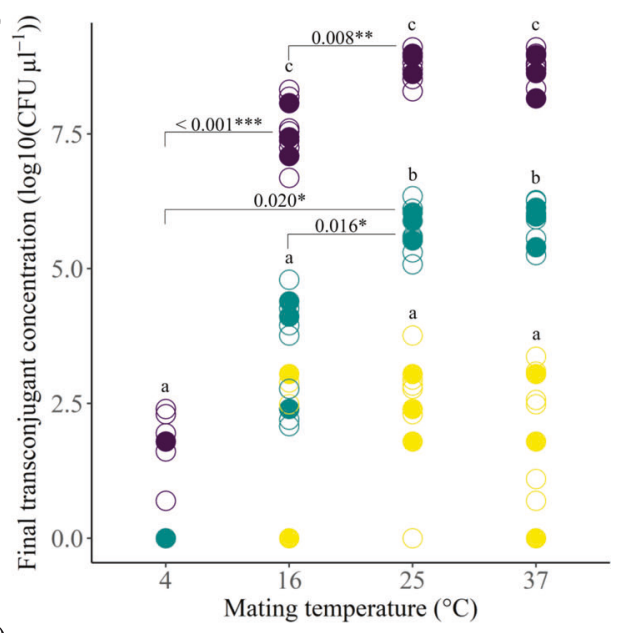

(D)

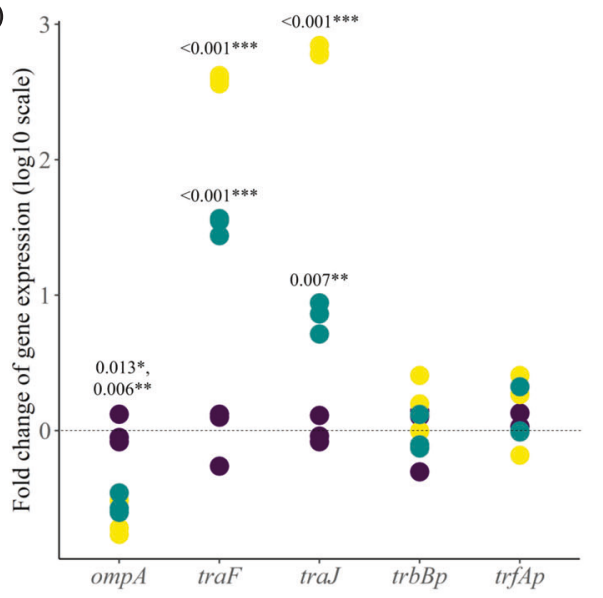

(E) Non-evaporative (NEV)

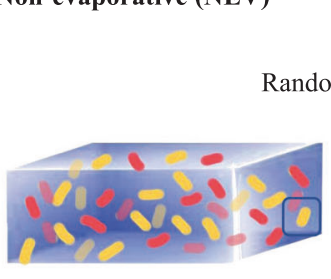

tion
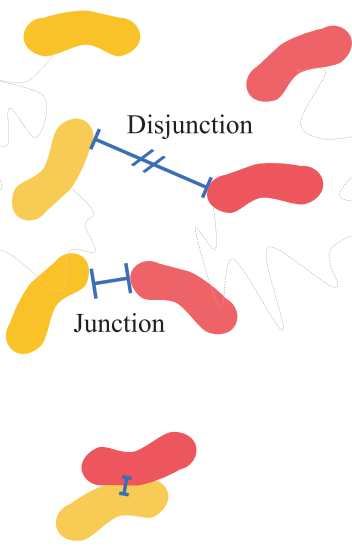

Junction with short distances

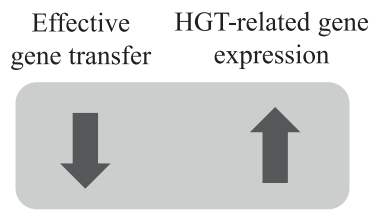

(F) Evaporative

Coffee ring effect (EV)

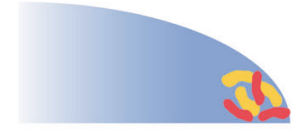

Coffee ring effect (EV)

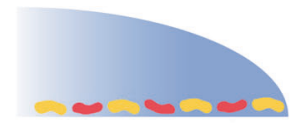

Marangoni convection (MC)

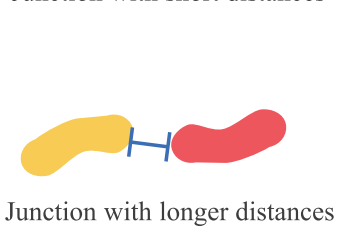

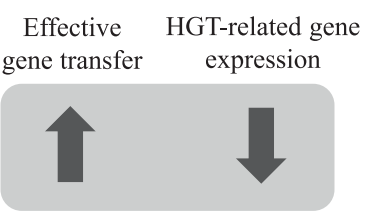

Effective HGT-related gene gene transfer expression

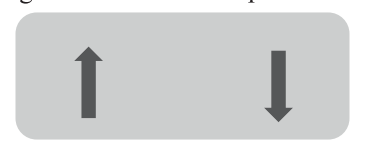

Supplementary Tables S2 and S3). We first quantified the effects of initial cell number, mating time, and mating temperature on RP4 conjugation (Fig. 2A and Supplementary Fig. S2), finding that the extent of RP4 conjugation increased with increasing initial cell number $\left(F_{6,36}=71.90\right.$, $P<0.001$; Supplementary Fig. S2A) and with mating temperature $\left(F_{6,36}=15.71, P<0.001\right.$; Fig. $2 \mathrm{~B}$ and Supplementary Fig. S2B). The extent of RP4 conjugation was significantly greater under EV than MC conditions already after $2 \mathrm{~h}$ mating time (Fig. 2C), while the extent of RP4 conjugation after $8 \mathrm{~h}$ mating time was 1000 times greater under MC than NEV conditions (Fig. 2C). Finally, the extent of RP4 conjugation after $10 \mathrm{~h}$ mating time remained approximately constant, being $\sim 10^{6}$ times greater under EV than NEV conditions and 1500 times greater under MC than 
Fig. 2 Experimentally measured effects of environmental conditions (evaporative, EV; Marangoni convection, MC; non-evaporative, NEV) on the extent of RP4 conjugation and the relative expression levels of conjugation-related genes. The effects of $(A)$ the initial cell number, (B) mating temperature, and (C) mating time. For the initial cell number and mating temperature $(\mathbf{A}$ and $\mathbf{B})$, experimental replicates $(n=3)$ are depicted as filled circles and analytical replicates $(n=3$ per experimental replicate) are depicted as open circles. Some datapoints are not visible due to overlap with other datapoints. Statistically significant $(\leq 0.05)$ Holm-Bonferroni adjusted $P$ values are reported for relevant factor level comparisons (among levels of initial cell number or mating temperature within each evaporation condition). For all data (A, B, C), statistically significant differences between evaporation conditions are depicted with lower-case letters. D Relative expression levels of genes encoding conjugation-related membrane proteins (ompA), involved in mating pair formation (trbBp and traF) and involved with plasmid transfer and replication (trfAp and traJ). Expression levels are relative to those measured for EV conditions. Statistically significant ( $\leq 0.05)$ Holm-Bonferroni adjusted $P$ values are reported for comparisons between the gene expression levels of the MC and NEV treatments against the EV treatment. The degree of statistical significance is indicated with *, ** or *** for $P$ values that are $0.05-0.01,0.01-0.001$ or $<0.001$, respectively. Differences in the spatial distributions of cells and their cell-cell interactions under (E) non-evaporative and (F) evaporative conditions. Dotted lines in (E) represent bacterial trajectories of random motion and arrow thickness represents the magnitude of effective plasmid conjugation or conjugation-related gene expression.

NEV conditions (Fig. 2A). These outcomes are consistent with our expectations, where the number and duration of cell-cell contacts are the highest under EV conditions leading to a higher extent of RP4 conjugation.

We finally quantified the expression of conjugation-related genes using qPCR (see details in the Supplementary Information). The membrane protein-encoding ompA gene, which can modulate conjugation rates [13], was expressed at significantly higher levels under EV and MC than NEV conditions $\left(F_{2,6}=31.72, P<\right.$ 0.001 ; Fig. 2D and Supplementary Table S4). This agrees with a previous study demonstrating a positive relationship between ompA expression and conjugation rates [14]. However, genes involved with mating pair formation (trbBp and traF) $[15,16]$ and plasmid transfer and replication (trfAp and traJ) $[17,18]$ were not expressed or expressed at significantly lower levels under EV than NEV conditions (traF $F_{2,6}=1170.4, P<0.001$; traJ $F_{2,6}=1453.5, P<$ 0.001 ) (Fig. 2D and Supplementary Table S4). This contrasts with the common view that the extent of conjugative plasmid transfer is positively correlated with the expression of conjugation-related genes $[14,19]$ (although empirical evidence for such correlations is rare and may not exist for a multitude of dynamical reasons [20]). Mating pair formation and plasmid transfer and replication machinery are required to establish cell-cell junctions, replicate plasmids, and transfer plasmids to recipient cells $[5,8]$. Under NEV conditions, cells can actively move and are unlikely to be collected at a fixed location (Supplementary Video 3). Such movements not only minimize the probability of forming cell-cell junctions but can also destabilize cell-cell junctions, thus requiring higher expression levels of these genes to achieve successful conjugation (Fig. 2E). In contrast, under EV conditions evaporation-induced passive cell movement results in larger numbers of cells pinned on surfaces or trapped at an air-water-solid interface. This results in a highdensity biofilm-like mass with increased numbers and durations of cell-cell contacts. This, in turn, stabilizes cell-cell junctions and increases conjugation efficiency, thus requiring lower expression levels of these genes to achieve successful conjugation (Fig. 2F).

In conclusion, evaporation-induced passive cell movement can modulate the spatial distributions of cells with profound effects on the extent of plasmid conjugation. Our results demonstrate that evaporation-induced hydrodynamics can be an important determinant of plasmid transfer and should therefore be considered when predicting plasmid fate and designing strategies to control plasmid proliferation. Our results are potentially generalizable to a wide variety of conjugative plasmids and microbial communities, including those important for human health, the environment, and biotechnology.

\section{REFERENCES}

1. Partridge SR, Kwong SM, Firth N, Jensen SO. Mobile genetic elements associated with antimicrobial resistance. Clin Microbiol Rev. 2018;31:e00088-17.
2. Pilla G, Tang CM. Going around in circles: virulence plasmids in enteric pathogens. Nat Rev Microbiol. 2018;16:484-95.

3. Sayler GS, Hooper SW, Layton AC, Henry King JM. Catabolic plasmids of environmental and ecological significance. Microbiol Ecol. 1990;1:1-20.

4. Timmis KN, Steffan RJ, Unterman R. Designing microorganisms for the treatment of toxic waste. Annu Rev Microbiol. 1994;48:525-57.

5. Hayes CS, Aoki SK, Low DA. Bacterial contact-dependent delivery systems. Annu Rev Genet. 2010;44:71-90.

6. Nellimoottil TT, Rao PN, Ghosh SS, Chattopadhyay A. Evaporation-induced patterns from droplets containing motile and nonmotile bacteria. Langmuir. 2007;23:8655-8.

7. Unc A, Goss MJ. Movement of faecal bacteria through the vadose zone. Water Air Soil Pollut. 2003;149:327-37.

8. Samuels AL, Lanka E, Davies JE. Conjugative junctions in RP4-mediated mating of Escherichia coli. J Bacteriol. 2000;182:2709-15.

9. Rhee MS, Kim JW, Qian Y, Ingram LO, Shanmugam KT. Development of a plasmid vector and electroporation condition for gene transfer in sporogenic lactic acid bacterium, Bacillus coagulans. Plasmid. 2007;58:13-22.

10. Deegan RD, Bakajin O, Dupont TF, Huber G, Nagel SR, Witten TA. Capillary flow as the cause of ring stains from dried liquid drops. Nature. 1997;389:827-9.

11. Scriven LE, Sternling CV. The Marangoni effects. Nature. 1960;187:186-8.

12. Seo C, Jang D, Chae J, Shin S. Altering the coffee-ring effect by adding a surfactant-like viscous polymer solution. Sci Rep. 2017;7:500.

13. Sun D, Wang B, Zhu L, Chen M, Zhan L. Block and boost DNA transfer: opposite roles of OmpA in natural and artificial transformation of Escherichia coli. PLoS ONE. 2013;8:e59019.

14. Qiu Z, Yu Y, Chen Z, Jin M, Yang D, Zhao Z, et al. Nanoalumina promotes the horizontal transfer of multiresistance genes mediated by plasmids across genera. Proc Natl Acad Sci USA. 2012;109:4944-9.

15. Haase J, Lurz R, Grahn AM, Bamford DH, Lanka E. Bacterial conjugation mediated by plasmid RP4: RSF1010 mobilization, donor-specific phage propagation, and pilus production require the same Tra2 core components of a proposed DNA transport complex. J Bacteriol. 1995;177:4779-91.

16. Pansegrau W, Lanka E, Barth PT, Figurski DH, Guiney DG, Haas D, et al. Complete nucleotide sequence of Birmingham IncP alpha plasmids. Compilation and comparative analysis. J Mol Biol. 1994;239:623-63.

17. Schröder G, Lanka $E$. The mating pair formation system of conjugative plasmids-A versatile secretion machinery for transfer of proteins and DNA. Plasmid. 2005;54:1-25.

18. Ziegelin G, Fürste JP, Lanka E. TraJ protein of plasmid RP4 binds to a 19-base pair invert sequence repetition within the transfer origin. J Biol Chem. 1989;264:11989-94.

19. Zhang Y, Gu AZ, He M, Li D, Chen J. Subinhibitory concentrations of disinfectants promote the horizontal transfer of multidrug resistance genes within and across Genera. Environ Sci Technol. 2016;51:570-80.

20. Rocca JD, Hall EK, Lennon JT, Evans SE, Waldrop MP, Cotner JB, et al. Relationships between protein-encoding gene abundance and corresponding process are commonly assumed yet rarely observed. ISME J. 2015;9:1693-9.

\section{ACKNOWLEDGEMENTS}

We thank Professor Junwen Li for providing E. coli HB101 and Jiangsu Provincial Key Lab for Organic Solid Waste Utilization (Nanjing Agricultural University) for providing E. coli K12. We thank Dr Wenjuan Zheng and Mr Hanqing Wu for their helpful discussions. We thank Miao Han for her excellent technical assistance. CR, GC, and GW were supported by grants from the National Natural Science Foundation of China (41877412), the 2115 Talent Development Program of China Agricultural University, and the Scholarship of the National Thousand 
(Young) Talents Program' of China. JR was supported by a Discretionary Funds (Category: SEED) grant from Eawag.

\section{AUTHOR CONTRIBUTIONS}

$C R$ and GW conceived the research question and designed the methodology and experiments. CR performed the experiments. JR performed the statistical analyses. All authors analyzed and interpreted the data. All authors wrote the paper. All authors reviewed and approved the final version of the paper. GW initiated the project and DRJ and GW coordinated it.

\section{COMPETING INTERESTS}

The authors declare no competing interests.

\section{ADDITIONAL INFORMATION}

Supplementary information The online version contains supplementary material available at https://doi.org/10.1038/s43705-021-00057-5.

Correspondence and requests for materials should be addressed to David $\mathrm{R}$. Johnson or Gang Wang.
Reprints and permission information is available at http://www.nature.com/ reprints

Publisher's note Springer Nature remains neutral with regard to jurisdictional claims in published maps and institutional affiliations.

(i) Open Access This article is licensed under a Creative Commons Attribution 4.0 International License, which permits use, sharing, adaptation, distribution and reproduction in any medium or format, as long as you give appropriate credit to the original author(s) and the source, provide a link to the Creative Commons license, and indicate if changes were made. The images or other third party material in this article are included in the article's Creative Commons license, unless indicated otherwise in a credit line to the material. If material is not included in the article's Creative Commons license and your intended use is not permitted by statutory regulation or exceeds the permitted use, you will need to obtain permission directly from the copyright holder. To view a copy of this license, visit http://creativecommons. org/licenses/by/4.0/.

(c) The Author(s) 2021 